\title{
Tendência temporal e distribuição espacial da sífilis congênita no estado do Rio Grande do Sul entre 2001 e 2012
}

\author{
Temporal trend and spatial distribution of congenital syphilis \\ in the state of Rio Grande do Sul between 2001 and 2012
}

Lisiane Ortiz Teixeira ${ }^{1}$

Vanusa Belarmino ${ }^{1}$

Carla Vitola Gonçalves ${ }^{1}$

Raúl Andrés Mendoza-Sassi ${ }^{1}$
${ }^{1}$ Faculdade de Medicina, Universidade Federal do Rio Grande. R. Visconde de Paranaguá 102, Centro. 96203-900 Rio Grande RS Brasil. lisiane.teixeira@furg.br

\begin{abstract}
The scope of the study was to evaluate the temporal trend and spatial distribution of congenital syphilis (CS) in the state of Rio Grande do Sul. All cases reported by the SINASC/DATASUS between 2001 and 2012 were included. The number of live births was obtained from DATASUS. Incidence rates of CS were grouped according to micro-regions of IBGE and analyzed for the entire period and for triennia. The spatial correlation was analyzed by the global Moran index (I) and the local index. 3,613 cases were reported. Between 2007 and 201289 neonates (3.6\%) died. Rates varied from to 1.03 in 2001 to 5.1 cases per 1000 live births in 2012, with an annual increase of 0.84 cases per 1000 live births $(p<0.01)$ and $93.88 \%$ of explained variance. The micro-regions were spatially independent $(I=0.06 ; p=0.25)$, with Porto Alegre having the highest incidence (4.19 cases / 1000 live births) and Jaguarão the lowest (0.23 cases / 1000 live births). Micro-regions with significant local spatial dependence were observed. The increase in cases of CS highlights poor prenatal quality care. Identification of the micro-regions with the highest incidence is essential to focus public policy on this health problem.
\end{abstract}

Key words Spatial analysis, Time-series studies, Congenital syphilis, Vertical infectious disease transmission, Epidemiological surveillance
Resumo Objetivou-se avaliar a tendência temporal e a distribuição espacial da Sífilis Congênita (SC) no estado do Rio Grande do Sul. Todos os casos notificados pelo Sistema Nacional de Agravos de Notificação entre 2001 e 2012 foram incluídos. Os números de nascidos vivos foram obtidos do SINASC/DATASUS. As taxas de incidência de SC foram agrupados conforme as microrregiões do IBGE e analisados para todo o periodo e em triênios. A correlação espacial foi analisada pelo indice de Moran global (I) e local. Foram notificados 3.613 casos. Entre 2007 e 2012 morreram 89 neonatos (3,6\%). As taxas de SC variaram de 1,03 em 2001 a 5,1 casos por 1.000 nascidos vivos em 2012, com um incremento anual de 0,84 casos por 1.000 nascidos vivos $(p<0,01)$ e 93,88\% da variação explicada. As microrregiões foram espacialmente independentes $(I=0,06 ; p=0,25)$, tendo Porto Alegre a maior incidência (4,19 casos/1.000 nascidos vivos) e Jaguarão a menor (0,23 casos/1.000 nascidos vivos). Observaram-se microrregiões com dependência espacial local significativa. O aumento dos casos de SC salienta um déficit na qualidade do pré-natal. A identificação das microrregiões com maior incidência é essencial para focalizar as politicas públicas sobre esse tema.

Palavras-chave Análise espacial, Estudos de séries temporais, Siffilis congênita, Transmissão vertical de doença infecciosa, Vigilância epidemiológica 


\section{Introdução}

A sífilis é um grave problema de saúde pública no mundo, mesmo que na maioria dos casos o tratamento apresente um desenlace satisfatório' ${ }^{1}$. A Sífilis Congênita é a transmissão do Treponema pallidum por via transplacentária da gestante infectada para o recém-nascido, podendo ocorrer em qualquer fase da gravidez ${ }^{2}$ e é causa frequente de morbidade perinatal, além de provocar aborto espontâneo, natimorto ou morte perinatal em cerca de $40 \%$ dos fetos de gestantes não tratadas ${ }^{3}$.

No Brasil, para o período de 2010 a 2011, o Ministério da Saúde realizou um estudo com 36 mil parturientes e estimou uma prevalência de sífilis em gestantes de $0,85 \%$ para o país e de $0,48 \%$ para a região $\mathrm{Sul}^{4}$. Nas gestantes não tratadas, a taxa de transmissão vertical da sífilis é de $70 \%$ a $100 \%$ nas fases primária e secundária da doença, reduzindo para $30 \%$ nas fases tardias da infecção materna (latente tardia e terciária) ${ }^{2}$. A estimativa é a de que nasçam, anualmente, 12 mil neonatos com Sífilis Congênita, sendo que 50\% desses são assintomáticos ${ }^{3}$.

A Sífilis Congênita no Brasil está na lista de doenças de notificação compulsória desde 1986 e a Sífilis na Gestação desde $2005^{2}$, na tentativa de facilitar e ampliar o diagnóstico, além de garantir o tratamento adequado ${ }^{1}$. Em 2006 foi aprovado o Pacto pela Saúde que tem como prioridade reduzir a mortalidade materna e infantil controlando as taxas de transmissão vertical da Sífilis e do HIV ${ }^{4}$. Estudos sobre a tendência temporal e a distribuição espacial permitem uma maior compreensão sobre as regiões que carecem de maior atenção, além de desempenharem um importante papel sobre o planejamento e o impacto dos programas derivados das políticas públicas vigentes. Neste contexto, este estudo teve como objetivo avaliar a tendência temporal e a distribuição espacial da Sífilis Congênita no estado do Rio Grande do Sul (RS) entre os anos de 2001 a 2012.

\section{Métodos}

Foi realizado um estudo epidemiológico observacional do tipo ecológico. A população em risco considerada foi a de nascidos vivos no estado do Rio Grande do Sul de acordo com o Sistema de Informação sobre os Nascidos Vivos (SINASC), disponível no site do Banco de Dados do Sistema único de Saúde (DATASUS) 5 .

Os casos foram os notificados e confirmados pelo Sistema Nacional de Agravos de Notifica- ção (SINAN/DATASUS) ${ }^{6,7}$ para o estado do Rio Grande do Sul entre os anos de 2001 a 2012. Segundo o DATASUS ${ }^{8}$, é considerado caso de sífilis congênita:

- toda criança ou aborto ou natimorto de mãe com evidência clínica para sífilis e/ou com sorologia não treponêmica reagente para sífilis com qualquer titulação, na ausência de teste confirmatório treponêmico, realizada no pré-natal ou no momento do parto ou curetagem que não tenha sido tratada ou recebido tratamento inadequado;

- todo indivíduo com menos de 13 anos com as seguintes evidências sorológicas: titulações ascendentes (testes não treponêmicos); e/ou o testes não treponêmicos reagentes após 6 meses de idade (exceto em situação de seguimento terapêutico); e/ou o testes treponêmicos reagentes após 18 meses de idade; e/ou títulos em teste não treponêmico maiores que os da mãe;

- todo indivíduo com menos de 13 anos com teste não treponêmico reagente e evidência clínica ou liquórica ou radiológica de sífilis congênita e

- toda situação de evidência de T. pallidum em placenta ou cordão umbilical e/ou amostra de lesão, biópsia ou autópsia de criança, aborto ou natimorto.

Foi escolhido o ano de 2001 para o início da análise por ser o primeiro ano com dados disponível no sistema. Até a realização deste estudo, o ano de 2012 era o último ano com dados completos, sendo que para 2013, 2014 e 2015 os dados preliminares ainda seriam atualizados.

Os dados relacionados à Sífilis na Gestação de 2005 a $2006^{9}$ e 2007 a $2012^{10}$ também foram obtidos a partir do SINAN/DATASUS. Informações sobre a Sífilis na Gestação de 2001 a 2004 não foram coletados visto que a notificação compulsória para essa doença foi estabelecida apenas a partir do ano de 2005. O número de equipes da Estratégia Saúde da Família (ESF) de 2001 a 20012 para o estado do Rio Grande do Sul foi obtido do site do Departamento de Atenção Básica $(\mathrm{DAB})^{11}$.

Foram calculadas as taxas de incidência de Sífilis Congênita e da detecção da Sífilis na Gestação para cada ano do estudo, utilizando as seguintes fórmulas":

No de casos novos confirmados de Sífilis

Congênita ocorridos no estado do Rio

Grande do Sul em determinado período $\mathrm{X} 1.000$

No de recém-nascido no Rio Grande

do Sul durante o mesmo período 
No de casos novos confirmados de Sífilis na Gestação ocorridos no estado do Rio Grande do Sul em determinado período

No de recém-nascido no Rio Grande X 1.000

do Sul durante o mesmo período

Foram calculados os coeficientes de correlação de Spearman $(\rho)$ entre o número de ESF e a taxa de incidência de Sífilis Congênita e entre o número de ESF e a taxa de detecção de Sífilis em Gestantes. Para avaliar a relação entre os anos e as taxas de incidência de Sífilis Congênita e de detecção de Sífilis em Gestantes foram realizadas regressões múltiplas, considerando o número de ESF como covariável. O programa utilizado foi o Stata, versão $13^{{ }^{112}}$.

A taxa de transmissão vertical entre os anos de 2008 a 2012 foi calculada pela seguinte fórmula $^{13}$ :

No de casos notificados de Sífilis Congênita ocorridos no estado do Rio Grande do Sul em determinado período

No de casos notificados de Sífilis em X 100

gestantes ocorridos no Rio Grande do Sul durante o mesmo período

O número de óbitos por Sífilis Congênita está disponível no SINAN/DATASUS a partir do ano de 2007, sendo a taxa de letalidade calculada conforme a seguinte fórmula ${ }^{14}$ :

No confirmado de óbitos causados pela Sífilis Congênita ocorridos no estado do Rio Grande do Sul em determinado período

No confirmado de casos de Sífilis X 100

Congênita ocorridos no Rio Grande do Sul durante o mesmo período

Com a finalidade de descrever a população do estudo, foram coletadas, para cada ano, informações sobre as características das mães e dos neonatos presentes no site do $\operatorname{SINAN}^{6,7}$. Em seguida, foi feita uma média aritmética para cada uma das características entre os anos de 2001 a 2012. Os dados analisados das mães foram faixa etária, cor da pele, escolaridade, zona de residência, realização do pré-natal, momento do diagnóstico da Sífilis Materna e tratamento do parceiro. Para o recém-nascido notificado as variáveis foram faixa etária, sexo, classificação final da doença (Sífilis Congênita Recente, Sífilis Congênita Tardia, Natimorto Sifilítico, Aborto por sífilis) e evolução (vivo, óbito pelo agravo notificado, óbito por outra causa). Os dados "Ignorados" ou "Em Branco" não foram excluídos a fim de evitar a subestimação dos dados e foram analisados como uma única categoria para cada uma dessas variáveis.

Para a análise espacial das taxas de incidência de Sífilis Congênita, as unidades de análise foram as microrregiões estabelecidas pelo Instituto Brasileiro de Geografia e Estatística (IBGE) ${ }^{15}$ conforme apresentado na Figura 1. Os dados foram subdivididos em quatro triênios: 2001/2002/2003; 2004/2005/2006; 2007/2008/2009 e 2010/2011/2012, sendo também estudado o período total de análise, de 2001 a 2012. A decisão de utilizar triênios foi escolhida para atenuar as oscilações anuais dos dados ${ }^{16,17}$. A malha digital das microrregiões foi obtida no site do Instituto de Pesquisa Econômica Aplicada (IPEA) ${ }^{18}$. A população em risco considerada foi a de nascidos vivos para cada microrregião, obtida no site DATASUS ${ }^{5}$.

Foi realizada a construção e a análise de mapas das taxas de incidência de Sífilis Congênita para cada microrregião em cada triênio e para os 12 anos de análise. O intervalo das taxas de incidência de Sífilis Congênita presente nos mapas foi definido a partir do intervalo estipulado no triênio 2001/2002/2003, com o objetivo de comparar os resultados entre os diferentes triênios e para todo período. Posteriormente, foram calculadas as autocorrelações espaciais para análise da dependência espacial entre as taxa de incidência de Sífilis Congênita e cada microrregião em cada período. Foram calculados os Índices Globais de Associação Espacial (Índice de Moran (I)) e os Îndices Locais de Associação Espacial (LISA) com os seus respectivos p-valores. Esses índices variam de $-1 \mathrm{a}+1$, sendo que valores positivos e negativos possuem autocorrelação espacial positiva e negativa, respectivamente ${ }^{19}$. Os valores próximos de zero indicam inexistência de autocorrelação espacial, isto é, os eventos são aleatórios $^{19}$. Em contrapartida, valores mais próximo de 1 significam uma maior semelhança entre vizinhos $^{19}$. O nível de significância adotado para as análises foi de $1 \%{ }^{16}$. A autocorrelação local foi realizada para identificar as regiões que apresentaram correlação local significativamente diferente do observado no resto dos dados ${ }^{16,19}$. Após essa análise, as microrregiões foram classificadas em sem significância, com significância de 95\% (1,96 sd), com significância de 99\% (2,54 sd) e com significância de 99,9\% (3,2 sd $)^{19}$. 


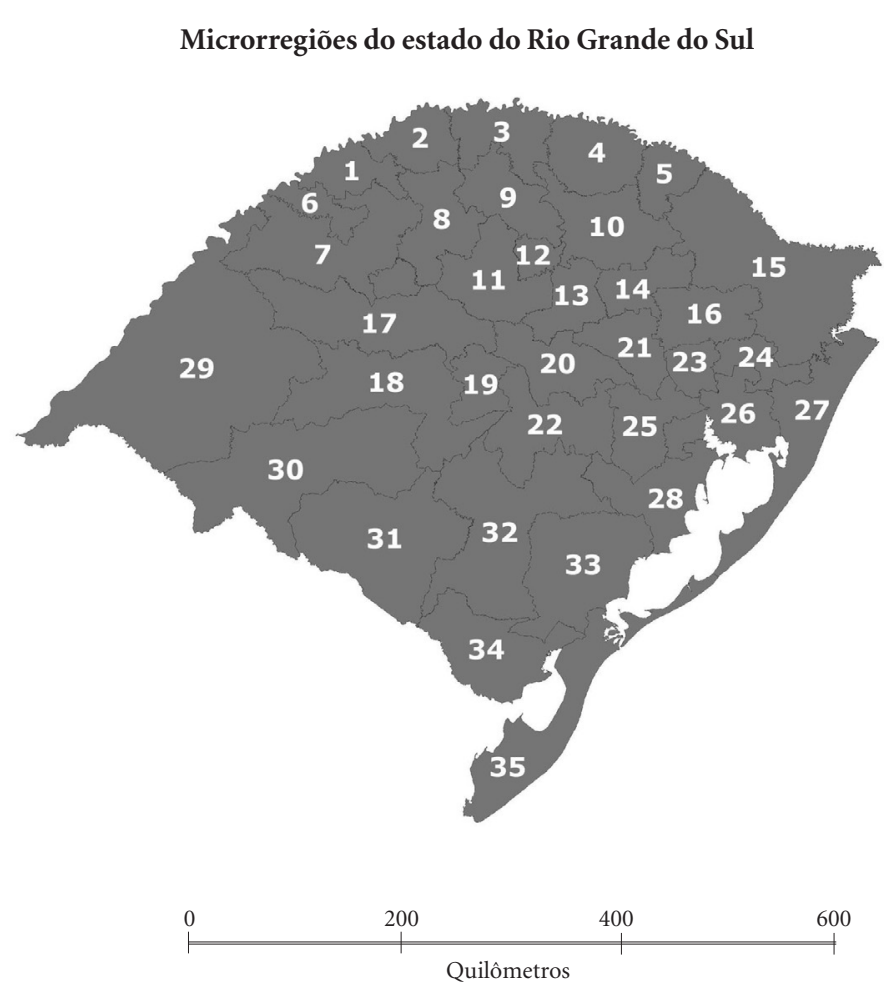

$$
\begin{aligned}
& 1 \text { - Santa Rosa } \\
& \text { 2 - Três Passos } \\
& \text { 3 - Frederico Westphalen } \\
& \text { 4 - Erechim } \\
& \text { 5 - Sanaduva } \\
& \text { 6 - Cerro Largo } \\
& \text { 7 - Santo Ângelo } \\
& \text { 8 - Ijuí } \\
& 9 \text { - Carazinho } \\
& 10 \text { - Passo Fundo } \\
& 11 \text { - Cruz Alta } \\
& 12 \text { - Não-Me-Toque } \\
& 13 \text { - Soledade } \\
& 14 \text { - Guaporé } \\
& 15 \text { - Vacaria } \\
& 16 \text { - Caxias do Sul } \\
& 17 \text { - Santiago } \\
& 18 \text { - Santa Maria } \\
& 19 \text { - Restinga Seca } \\
& 20 \text { - Santa Cruz do Sul } \\
& 21 \text { - Lajeado-Estrela } \\
& 22 \text { - Cachoeira do Sul } \\
& 23 \text { - Montenegro } \\
& 24 \text { - Gramado-Canela } \\
& 25 \text { - São Jerônimo } \\
& 26 \text { - Porto Alegre } \\
& 27 \text { - Osório } \\
& 28 \text { - Camaquã } \\
& 29 \text { - Campanha Central } \\
& 30 \text { - Campanha Meridional } \\
& 32 \text { - Serras do Sudeste } \\
& 33 \text { - Pelotas } \\
& 34 \text { - Jaguarão } \\
& 35 \text { - Litoral Lagunar } \\
&
\end{aligned}
$$

Figura 1. Estado do Rio Grande do Sul dividido em Microrregiões conforme Instituto Brasileiro de Geografia e Estatística (IBGE).

A fim de detectar as áreas com maior, menor e intermediários valores das taxas de incidência de Sífilis Congênita, foram construídos mapas conforme os quadrantes do diagrama de espalhamento de Moran (BoxMap) ${ }^{16}$. No quadrante um (alta/alta), microrregiões com alta taxa de incidência e vizinhos também com alta taxa; no quadrante dois (baixa/baixa), microrregiões com baixa taxa de incidência e vizinhos também com baixa taxa; no quadrante três (alta/baixa), microrregiões com alta taxa e vizinhos com baixa taxa e o quadrante quatro (baixa/alta), microrregiões com baixa taxa e vizinhos com alta taxa de incidência ${ }^{19}$. A associação espacial nos dois primeiros quadrantes é positiva, com microrregiões e seus vizinhos com valores semelhantes. Em contrapartida, nos quadrantes três e quatro a associação espacial é negativa, com microrregiões e seus vizinhos com valores diferentes. Os mapas, assim como a análise de autocorrelação, foram obtidos no programa TerraView versão 3.2.120.

Os dados analisados, que foram coletados exclusivamente de fontes secundárias conforme descrito acima, estão disponíveis para todos os interessados sem nenhum tipo de limitação. Desse modo, por se tratar de base de dados públicos, não houve necessidade de encaminhar este trabalho para a aprovação do Comitê de Ética e de Pesquisa $^{16,21}$. Assim como em outros estudos ecológicos similares ${ }^{22-24}$, o anonimato dos indivíduos foi assegurado.

\section{Resultados}

Ao longo da análise de 12 anos, 1.718 .651 crianças nasceram no estado do Rio Grande do Sul, com 3.613 casos notificados e confirmados de Sífilis Congênita. Em relação às características dos casos de Sífilis Congênita, 59,6\% das mães tinham cor da pele branca, 55,9\% tinham o ensino fundamental incompleto ou eram analfabetas; $50,8 \%$ compreendiam a faixa etária entre 20 e 29 anos e $93,5 \%$ residiam na zona urbana. A respeito do pré-natal, $77,4 \%$ das gestantes realizaram o acompanhamento, sendo que em $51,9 \%$ o teste para sífilis foi realizado durante esse período. No decorrer dos 12 anos de análise, foi constatado 
que uma parcela das mulheres $(0,6 \%)$ não realizou o exame para sífilis nem no pré-natal, na hora do parto/curetagem ou após o parto. O parceiro não foi tratado em $54,4 \%$ dos casos. No que tange os neonatos, $47,3 \%$ eram do sexo feminino; em $96,5 \%$ a doença apareceu até os seis dias de vida; em $95,8 \%$ a classificação final foi Sífilis Congênita Recente e em 89,0\% os casos estavam vivos no momento da notificação. Vale ressaltar que a opção "Em Branco" ou "Ignorado" foi observada em $15,1 \%$ para cor da pele; $20,6 \%$ para escolaridade; $3,7 \%$ para o pré-natal; $5,4 \%$ para o momento que as mulheres realizaram o teste; $30,4 \%$ para o tratamento do parceiro; $5,2 \%$ para a faixa etária da mãe; $2,9 \%$ para a zona de residência; $7,7 \%$ para o sexo do neonato; $14,1 \%$ para a classificação final e 3,4\% para a evolução final da doença (sobreviveu, óbito causado pela sífilis, óbito por outro motivo). A respeito do tempo para o aparecimento da doença, entre 2001 e 2006 não ocorreram casos "Em Branco" ou "Ignorado" e entre os anos de 2006 e 2012 essas opções não estavam mais disponíveis no sistema.

A taxa de incidência de Sífilis Congênita mais baixa foi de 0,78/1.000 nascidos vivos em 2002 e a mais alta foi 5,03/1.000 nascidos vivos em 2012 . $\mathrm{O}$ ano de 2010 apresentou a menor letalidade da doença $(0,7 \%)$ e o ano de 2012 a maior $(5,1 \%)$. Entre 2007 e 2012, vieram a óbito em decorrência da doença 89 neonatos, representando 3,6\% dos casos do período (Tabela 1). No modelo de regressão entre as taxas de incidência de Sífilis Congênita, a série de anos e o número de equipes da ESF, cada incremento em um ano determinou um aumento de 0,84 casos por mil nascidos vivos ( $\mathrm{p}<0,01)$ e o modelo explicou 93,88\% da variação (R2 ajustado $=0,9388)$. Para analisar se esse aumento detectado foi devido a um aumento nas notificações, estudou-se a correlação entre a taxa de incidência de Sífilis Congênita e o número de equipes da ESF implantadas (de 350 em 2001 a 1.548 equipes em 2012) e observou-se uma correlação fortemente positiva e significativa $(\rho=0$, 96; $\mathrm{p}<0,01)$.

As taxas de detecção de Sífilis em Gestantes variaram de 0,46/1.000 nascidos vivos em 2006 a 6,69/1.000 nascidos vivos em 2012 (Tabela 1). O modelo de regressão entre as taxas de detecção de Sífilis em Gestante, a série temporal e o número de equipes da ESF apresentou para cada incremento anual um aumento de 1,08 casos por mil nascidos vivos $(\mathrm{p}=0,367)$ e o modelo explicou $79,53 \%$ da variação $(\mathrm{R} 2$ ajustado $=0,7953)$. O coeficiente de correlação entre a taxa de detecção de Sífilis em Gestantes e o número de equipes da ESF implantadas, calculado com o objetivo de verificar se esse aumento também não é resultado do aumento de equipes ESF, foi forte, positivo e significativo $(\rho=0,90 ; p<0,01)$. Foi detectada subnotificação da sífilis em mulheres grávidas durante o período do estudo, já que nos anos de 2006 e 2009, o número de casos notificados de Sífilis Congênita foi maior do que o número notificado de Sífilis em Gestantes. A taxa de transmissão vertical variou de 68,6\% em 2008 a 91,2\% em 2011 (Tabela 1).

Tabela 1. Taxa de Detecção de Sífilis em Gestantes, Taxa de Incidência de Sífilis Congênita e Letalidade da Sífilis Congênita no estado do Rio Grande do Sul entre os anos de 2001 e 2012 ( N = 1.718.651).

\begin{tabular}{|c|c|c|c|c|c|c|c|c|}
\hline Ano & $\begin{array}{l}\text { Casos em } \\
\text { Gestantes }\end{array}$ & $\begin{array}{l}\text { Casos de } \\
\text { Sífilis } \\
\text { Congênita }\end{array}$ & $\begin{array}{c}\text { Taxa de } \\
\text { Transmissão } \\
\text { Vertical }\end{array}$ & $\begin{array}{l}\text { Nascidos } \\
\text { vivos }\end{array}$ & $\begin{array}{c}\text { Taxa de } \\
\text { Detecção } \\
\text { em } \\
\text { Gestantes }^{\mathrm{a}}\end{array}$ & $\begin{array}{c}\text { Taxa de } \\
\text { Incidência } \\
\text { de Sífilis } \\
\text { Congênita }\end{array}$ & $\begin{array}{l}\text { Óbitos } \\
\text { por Sífilis } \\
\text { Congênita }\end{array}$ & $\begin{array}{c}\text { Letalidade } \\
\text { por Sífilis } \\
\text { Congênita }\end{array}$ \\
\hline 2001 & $\mathrm{ND}^{\mathrm{b}}$ & 167 & - & 160.590 & - & 1,03 & $\mathrm{ND}^{\mathrm{b}}$ & - \\
\hline 2002 & $\mathrm{ND}^{\mathrm{b}}$ & 121 & - & 155.261 & - & 0,78 & $\mathrm{ND}^{\mathrm{b}}$ & - \\
\hline 2003 & $\mathrm{ND}^{\mathrm{b}}$ & 184 & - & 149.165 & - & 1,23 & $\mathrm{ND}^{\mathrm{b}}$ & - \\
\hline 2004 & $\mathrm{ND}^{\mathrm{b}}$ & 142 & - & 153.015 & - & 0,93 & $\mathrm{ND}^{\mathrm{b}}$ & - \\
\hline 2005 & 218 & 183 & $83,9 \%$ & 147.199 & 1,25 & 1,24 & $\mathrm{ND}^{\mathbf{b}}$ & - \\
\hline 2006 & 66 & 215 & $\mathrm{NA}^{\mathrm{c}}$ & 141.331 & 0,46 & 1,52 & $\mathrm{ND}^{\mathrm{b}}$ & - \\
\hline 2007 & 307 & 262 & $85,3 \%$ & 133.401 & 2,30 & 1,96 & 12 & $4,5 \%$ \\
\hline 2008 & 379 & 260 & $68,6 \%$ & 135.143 & 2,80 & 1,92 & 10 & $3,8 \%$ \\
\hline 2009 & 306 & 363 & $\mathrm{NA}^{\mathrm{c}}$ & 133.652 & 2,29 & 2,71 & 10 & $2,7 \%$ \\
\hline 2010 & 482 & 417 & $86,5 \%$ & 133.243 & 3,61 & 3,12 & 3 & $0,7 \%$ \\
\hline 2011 & 638 & 582 & $91,2 \%$ & 137.710 & 4,63 & 4,22 & 17 & $2,9 \%$ \\
\hline 2012 & 929 & 717 & $77,2 \%$ & 138.941 & 6,69 & 5,10 & 37 & $5,1 \%$ \\
\hline
\end{tabular}

aPor 1.000 nascidos vivo. ${ }^{\text {a ND }}=$ Dado Não Disponível naquele ano. ${ }^{\mathrm{N} A}=$ Não aplicável. O número de casos de sífilis em gestantes notificados no ano foi inferior ao número de casos de Sífilis Congênita notificados no mesmo ano. 
A análise dos mapas com a distribuição espacial das taxas de incidência de Sífilis Congênita para cada triênio (Figura 2) e para os 12 anos de estudo (Figura 3a) evidenciam um aumento das taxas em todos os períodos. Considerando o período de 2001 a 2012 (Figura 3a), a microrregião Porto Alegre apresentou a maior taxa de incidência ( 4,19 casos/ 1.000 nascidos vivos) e a microrregião Jaguarão a menor $(0,23$ casos $/ 1.000$ nascidos vivos). Esse aumento foi maior nos triênios 2007/2008/2009 e 2010/2011/2012. No último triênio (Figura 2d), a microrregião Porto Alegre apresentou a maior taxa de incidência encontrada no estudo (8,52 casos/1.000 nascidos vivos).

Os Índices Globais de Moran nos triênios 2001/2002/2003 $(\mathrm{I}=-0,06 ; \mathrm{p}=$ $0,28), 2004 / 2005 / 2006(\mathrm{I}=-0,01 ; \mathrm{p}=$ $0,41), 2007 / 2008 / 2009(\mathrm{I}=0,12 ; \mathrm{p}=0,15)$,
2010/2011/2012 ( $\mathrm{I}=0,06 ; \mathrm{p}=0,36)$ e no período de 2001 a $2012(\mathrm{I}=0,06 ; \mathrm{p}=0,25)$ mostram que as microrregiões foram espacialmente independentes, ou seja, não há presença de cluster espacial (não estacionariedade) em todo o estado. Foi observada dependência espacial local com significância de 95\% (Santa Rosa, Três Passos, Ijuí, Osório, Passo Fundo e São Jerônimo), 99\% de significância (Montenegro) e 99,9\% de significância (Porto Alegre). As outras microrregiões foram consideradas sem significância espacial local.

No BoxMap (Figura 3b), as áreas representadas na cor preta (alta/alta) indicam as microrregiões com alta taxa de incidência cercados por microrregiões que também possuem alta taxa de incidência, estando localizada em toda a Mesorregião Metropolitana (microrregiões Mon-
Figura 2a - Triênio 2001/2002/2003

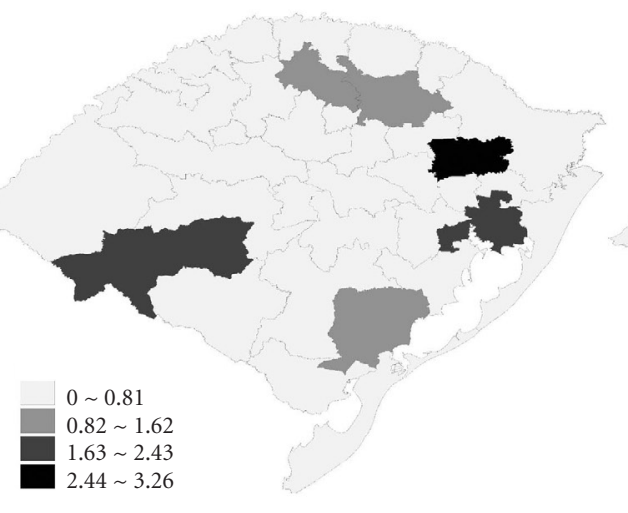

Figura 2c - Triênio 2007/2008/2009

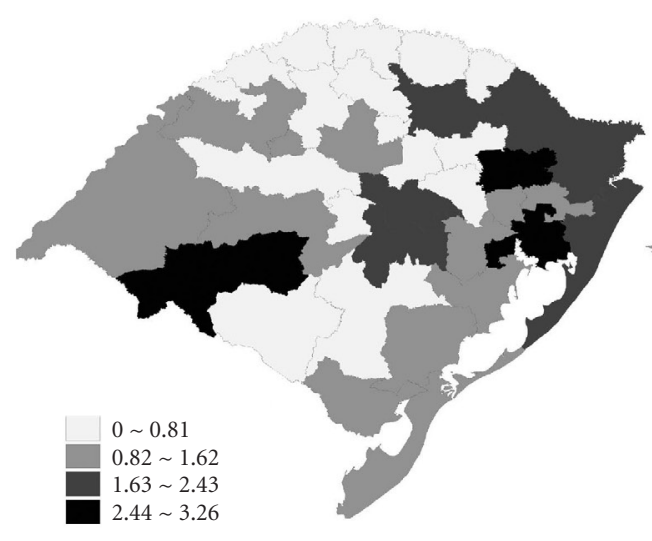

Figura 2b - Triênio 2004/2005/2006

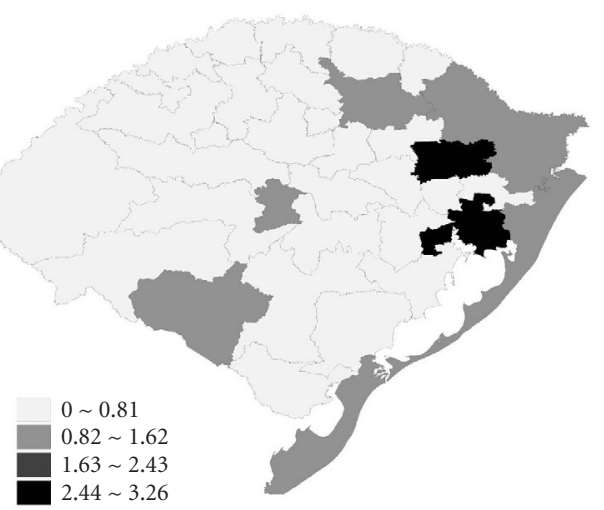

Figura 2d - Triênio 2010/2011/2012

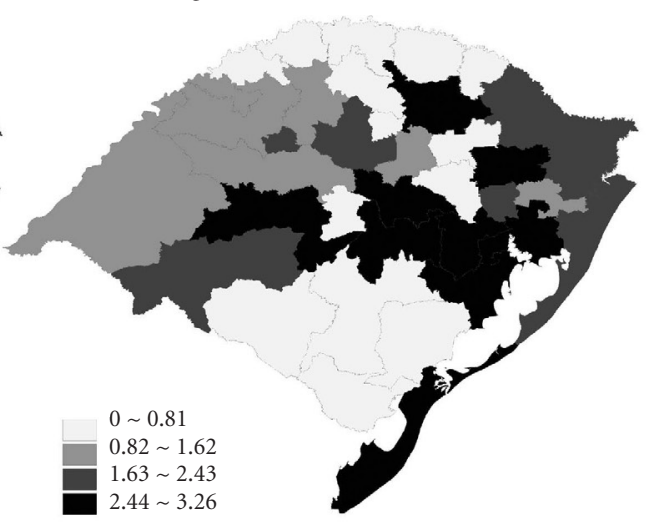

Figura 2. Distribuição Espacial trienal da Taxa de Incidência de Sífilis Congênita por 1.000 nascidos-vivos no Estado do Rio Grande do Sul no período de 2001 a 2012. 
Figura 3a - 2001 a 2012

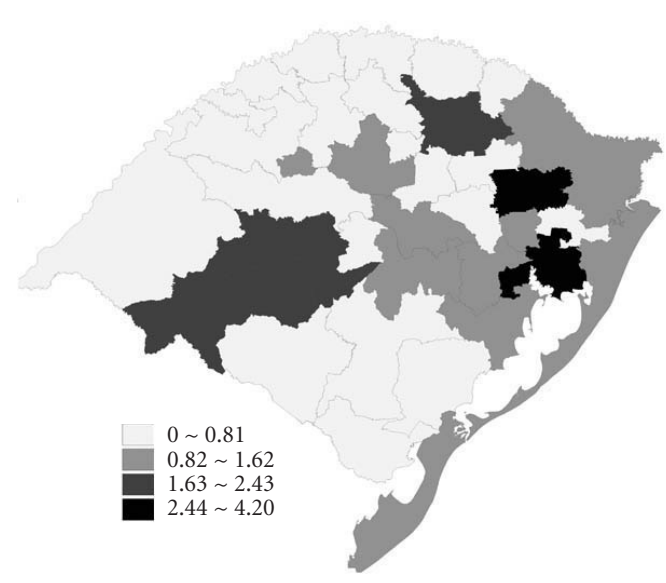

Figura 3b - BOXMap

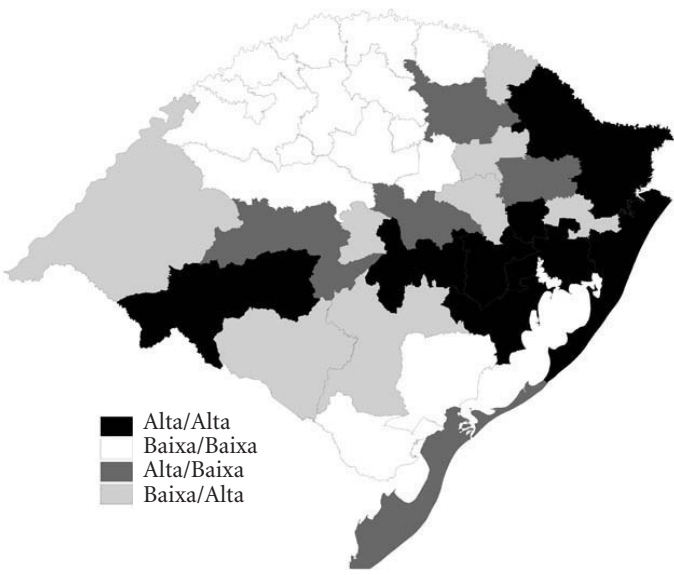

Figura 3. Taxa de Incidência de Sífilis Congênita por 1.000 nascidos-vivos no Estado do Rio Grande do Sul de 2001 a 2012 (esquerda) e BOXMAP da interação dessas taxas entre as microrregiões (direita).

tenegro, Gramado-Canela, São Jerônimo, Porto Alegre, Osório e Camaquã) e nas microrregiões Cachoeira do Sul, Vacaria e Campanha Central. As microrregiões em branco (baixa/baixa) são microrregiões com baixa taxa de incidência, cercados por microrregiões na mesma situação e estão todas localizadas na Mesorregião Noroeste Rio-Grandense (microrregiões Santa Maria, Três Passos, Frederico Westphalen, Erechim, Sananduva, Cerro Largo, Santo Ângelo, Ijuí, Carazinho, Passo Fundo, Cruz Alta, Não Me-Toque e Soledade). As áreas em cinza médio e cinza claro (alta/ baixa e baixa/alta, respectivamente) representam as microrregiões cercadas por microrregiões vizinhas em situação diferente da sua.

\section{Discussão}

A presente investigação objetivou avaliar a tendência temporal e a distribuição espacial da Sífilis Congênita no Estado do Rio Grande do Sul entre os anos de 2001 e 2012. Apesar de o Pacto pela Saúde ter como prioridade o controle das taxas de transmissão vertical da Sífilis ${ }^{4}$, os casos de Sífilis Congênita no estado do Rio Grande do Sul estão aumentando significativamente ao logo dos anos. A meta da Organização Pan-Americana de Saúde ${ }^{25}$ e do Ministério da Saúde ${ }^{4}$ era reduzir a incidência para 0,5 casos por 1.000 nascidos vivos até o ano de 2015. As taxas de incidência encontradas em todos os anos foram maiores do que este valor, chegando a 5,1 por 1.000 nasci- dos vivos no ano de 2012. Esse resultado é similar ao 6,0 casos/1.000 nascidos vivos encontrado no município de Natal, no Rio Grande do Norte, entre 2004 e $2007^{1}$ e ao 4,7 casos/1.000 nascidos vivos estimado para o Brasil para o ano de $2013^{25}$. Outro estudo avaliou a incidência para o Estado do Espírito Santo entre os anos de 2000 e 2005 e encontrou taxas variando de 4,94 a 6,25 casos por 1.000 nascidos vivos ${ }^{26}$. Isso mostra o quanto a quantidade de casos notificados nas diferentes regiões brasileiras é superior à meta.

No que concerne às taxas de incidência de Sífilis Congênita em estudos internacionais, diversas pesquisas também observaram aumentos. Uma investigação realizada no México para o período de 1990 a 2009 encontrou um aumento de 51,6\% dos casos no quinquênio 2004 a 2009 quando comparado com o de 1990 a $1994^{27}$. Na Colômbia, a incidência aumentou de 2,15 casos em 2005 para 3,28 casos/1.000 nascidos vivos em $2011^{28}$. A Espanha apresentou um aumento modesto, de 0,000 casos em 2003 a 0,223 casos/ 1.000 nascidos vivos em $2007^{29}$. Em contrapartida, estudos de diferentes países relataram uma redução nos números de casos. Em Shenzhen, na China, a incidência diminui de 1,15 em 2002 para 0,10 casos/1.000 nascidos em $2011^{30}$. Declínio análogo foi observado em Trindade, onde a incidência reduziu-se de 6,0 em 2003 para aproximadamente 0,5 casos/1.000 nascidos vivos em 2009, virtualmente alcançando a meta preconizada ${ }^{31}$. Para o ano de 2009, Réunion Island, na França, apresen- 
tou uma incidência de 0,28 casos/1.000 nascidos $\operatorname{vivos}^{32}$. Semelhantemente, o Reino Unido e o Sul da Itália apresentaram taxas menores do que 0,02 casos por mil nascidos vivos durante todo o período de 2010 a $2015^{33}$ e de 1997 a $2009^{34}$, respectivamente. Nos Estados Unidos, o número de casos, a mortalidade e a morbidade por Sífilis Congênita estão diminuindo anualmente ${ }^{35,36}$, sendo que o ano de 2012 apresentou a menor quantidade de casos notificados desde as mudanças nos critérios de diagnósticos estabelecidas no ano de $1988^{36}$. Dessa forma, é possível concluir que as incidências encontradas para o Brasil e os seus diferentes estados não está somente acima da meta, mas também mais elevada do que as taxas observadas em outros países. No entanto, é importante destacar que a comparabilidade entre os estudos fica prejudicada devido aos diferentes critérios que definem a Sífilis Congênita. Enquanto alguns autores consideram os natimortos para o cálculo de incidência ${ }^{33}$, outros excluem esses $\operatorname{casos}^{29}$. Similarmente, a idade limite da criança para ser diagnosticada com Sífilis Congênita varia de $18^{30}$ a 24 meses $^{29}$.

O aumento da Sífilis Congênita detectado no presente estudo pode ser resultado da melhoria da notificação de casos ao longo dos 12 anos, devido à utilização do teste-rápido, aos avanços da vigilância epidemiológica, à capacitação dos profissionais da saúde e à ampliação da cobertura do pré-natal em decorrência da implantação das equipes da Estratégia Saúde da Família (ESF) 3,37. Isso é corroborado pela presente investigação, uma vez que foi observada uma correlação positiva forte e significativa entre o aumento das equipes ESF e as taxas de incidência de Sífilis Congênita e de detecção da Sífilis em Gestantes. Dessa forma, conjectura-se que o aumento das taxas de incidência de Sífilis Congênita não está relacionado ao crescimento dos números de casos e sim ao do diagnóstico e da notificação no período.

Apesar da importância das investigações sobre a transmissão vertical da sífilis, ainda são poucos os estudos no país sobre suas taxas, sendo que a maioria dos trabalhos tem como enfoque o $\mathrm{HIV}^{13}$. A maior taxa encontrada no presente estudo foi $91,2 \%$ em 2011 e a menor foi $68,6 \%$ no ano de 2008, similar aos 69,8\% encontrado em um município de Santa Catarina ${ }^{13}$. No entanto, é necessário ressaltar que o presente estudo detectou subnotificações de Sífilis em Gestantes em alguns anos. A subnotificação e o preenchimento incompleto ou em branco dos casos de Sífilis Congênita e Sífilis em Gestantes é um problema encontrado em diferentes estudos de diferentes regiões do país ${ }^{1,37,38}$. Apesar da ficha de notificação ter em anexo um manual de instrução, é possível que a clareza do material esteja dificultando o correto preenchimento da notificação ${ }^{37}$. O preenchimento de forma correta é essencial para o monitoramento da Sífilis nas Gestantes e da Sífilis Congênita, além da avaliação dos programas e políticas públicas. Na presente investigação optou-se por não excluir os dados classificados como "Em Branco" ou "Ignorado" a fim de evitar a subestimação dos resultados. Isso permitiu observar que, mesmo que todos os 30,4\% dos dados mal preenchidos para o tratamento do parceiro sejam de casos onde os mesmos foram tratados, a quantidade de parceiros medicamentados ainda seria menor do que 50\%. Da mesma forma, se todos os dados fossem devidamente preenchidos, os achados ainda seriam os mesmos explicitados anteriormente. Assim, a escolha de não eliminar esses dados reforça os resultados encontrados.

A identificação das microrregiões com maiores taxas de incidência é uma ferramenta essencial para a elaboração de políticas públicas e para os respectivos programas de controle da Sífilis Congênita. Analisando os 12 anos, as microrregiões de Porto Alegre e Caxias do Sul sempre apresentaram taxas elevadas seguidas das microrregiões Campanha Central, Santa Maria e Passo Fundo. Por isso, com o objetivo de controlar a transmissão vertical, essas microrregiões receberam uma atenção maior dos gestores e profissionais da saúde. Avaliando apenas os últimos três anos (2010/2011/2012), além das microrregiões citadas acima, as de Cachoeira do Sul, Litoral Lagunar, Santa Cruz do Sul e São Jerônimo também apresentaram taxas elevadas e não devem ser desconsideradas. É importante salientar que as microrregiões Caxias do Sul e Porto Alegre possuem altos valores de Índice de Desenvolvimento Humano Municipal (IDHM) ${ }^{39}$. O fato da taxa de incidência de Sífilis Congênita ser maior nessas regiões pode ser resultado de melhores condições de atendimento nas unidades de saúde, consequentemente, melhor acesso aos métodos diagnósticos ${ }^{40}$, e melhores taxas de notificações. Em contrapartida, das nove microrregiões que fazem fronteira com a Argentina e com o Uruguai, sete apresentaram baixas taxas de incidência de Sífilis Congênita no período de 2001 a 2012. Como mencionado acima, o IDHM tem um papel fundamental nas taxas de diagnóstico e notificação. A maioria dos municípios de fronteira possuem um IDHM considerado médio, o que reforça a hipótese que municípios com melhores condi- 
ções de atendimento nas unidades de saúde diagnosticam e notificam mais e municípios com menores condições diagnosticam e notificam menos casos. Além disso, é preciso destacar que as baixas taxas encontradas nas regiões de fronteira podem ser resultado do fluxo de gestantes entre o Brasil e esses países. Um dado que reforça isso é o fato de que no ano de 2009 o Brasil e o Uruguai regulamentaram o acesso a serviços de saúde nas áreas de fronteira ${ }^{41}$. Esse fluxo ocorre devido ao fato de que as maternidades que são referência localizada nos países vizinhos são geograficamente mais perto desses municípios de fronteira que as maternidades brasileiras de referência ${ }^{42}$. O município de Barra do Quaraí é um desses exemplos, onde no ano de 2011 a prefeitura desse município criou um convênio próprio com um hospital uruguaio, além do convênio federal explicitado acima, para a realização de partos e atendimentos neonatais ${ }^{42}$. Outra microrregião localizada na fronteira é a de Jaguarão, a qual apresentou a menor taxa de incidência de Sífilis Congênita detectada no presente estudo. Consequentemente, nessas regiões podem ocorrer subnotificações do número de nascimentos e/ou do número de casos notificados.

Esse estudo apresentou limitações, já que por utilizar dados secundários que podem estar subnotificados, as taxas de incidência, detecção e transmissão vertical podem ser maiores do que o explicitado. No entanto, o SINAN é um sistema oficial e amplamente utilizado em trabalhos técnicos e científicos ${ }^{40}$. Além disso, as políticas públicas são baseadas nos dados notificados e este estudo mostrou que esses números estão aumentando significativamente com o tempo no Estado do Rio Grande do Sul. Como os estudos ecológicos dispõem de informações sobre exposição e doença apenas em nível populacional, não foi possível avaliar as comorbidades de forma efetiva ${ }^{40}$. Por fim, a comparação dos achados da presente investigação com outros estudos é prejudicada devido à escassez de trabalhos que avaliem a distribuição espacial e temporal da Sífilis Congênita.

A Sífilis Congênita é um marcador da qualidade de assistência à saúde materno-infantil em razão da efetiva redução do risco de transmissão transplacentária, de sua relativa simplicidade diagnóstica e do fácil manejo clínico/terapêutico ${ }^{3}$. Assim, o aumento das taxas de incidência nas diferentes regiões do estado é indicativo de falha na atenção do pré-natal. As informações aqui apresentadas poderão ser úteis para planejar monitoramentos mais efetivos nessas áreas, a fim de que ocorra uma mudança no quadro epidemiológico. Estudos posteriores são necessários para avaliar a eficácia dos programas e dos protocolos vigentes.

\section{Colaboradores}

LO Teixeira e V Belarmino participaram da concepção do projeto, da coleta e análise dos dados, da redação do artigo e aprovaram a versão final. CV Gonçalves e RA Mendoza-Sassi participaram da concepção do projeto e da análise dos dados, colaboraram criticamente na redação do artigo e aprovaram a versão final. 


\section{Referências}

1. Holanda MTCG, Bareto MA, Machado KMM, Pereira RC. Perfil Epidemiológico da sífilis congênita no Município de Natal, Rio Grande do Norte - 2004 a 2007. Epidemiol Serv Saúde 2011; 20(2):203-212.

2. Brasil. Ministério da Saúde (MS). Diretrizes para o Controle da Sífilis Congênita: manual de bolso. $2^{\text {a }}$ ed. Brasília: MS; 2006. (Manuais; 24).

3. Brasil. Ministério da Saúde (MS). Atenção ao pré-natal de baixo risco. Brasília: MS; 2012. (Cadernos de Atenção Básica, 32) (Série A. Normas e Manuais Técnicos)

4. Brasil. Ministério da Saúde (MS). Boletim Epidemiológico de Sifilis 2015. Brasília: MS; 2015. Ano IV nº 01.

5. Brasil. Ministério da Saúde (MS). Departamento de Informações e Informática do Sistema Único de Saúde (Datasus). Informações de Saúde. Estatísticas vitais. Mortalidade e nascidos vivos: nascidos vivos desde 1994 - Rio Grande do Sul. [acessado 2014 Fev 14]. Disponível em: http://tabnet.datasus.gov.br/cgi/deftohtm. exe?sinasc/cnv/nvrs.def

6. Brasil. Ministério da Saúde (MS). Sistemas de Informação de Agravos de Notificação (SINAN) Sífilis Congênita: Casos confirmados de 2001 a 2006. [acessado 2014 Fev 14]. Disponível em: http://tabnet.datasus.gov. $\mathrm{br} / \mathrm{cgi} / \mathrm{deftohtm}$.exe?sinanwin/cnv/sifilisrs.def

7. Brasil. Ministério da Saúde (MS). Sistemas de Informação de Agravos de Notificação (SINAN). Sífilis Congênita: Casos confirmados de 2007 a 2012. [acessado 2014 Fev 14]. Disponível em: http://tabnet.datasus.gov.br/ cgi/deftohtm.exe?sinannet/cnv/sifilisrs.def

8. Brasil. Ministério da Saúde (MS). Departamento de Informações e Informática do Sistema Único de Saúde (Datasus). Incidência de Sífilis Congênita. [acessado 2016 Abr 23]. Disponível em: http://tabnet.datasus. gov.br/tabdata/livroidb/Com2007/Com_D0111.pdf

9. Brasil. Ministério da Saúde (MS). Sistemas de Informação de Agravos de Notificação (SINAN). Sifilis Em Gestantes: Casos confirmados e notificados de 2005 a 2006. [acessado 2014 Set 7]. Disponível em : http:// tabnet.datasus.gov.br/cgi/deftohtm.exe?sinanwin/cnv/ sifilisgestanters.def

10. Brasil. Ministério da Saúde (MS). Sistemas de Informação de Agravos de Notificação (SINAN). Sifilis Em Gestantes: Casos confirmados e notificados de 2007 a 2012. [acessado 2014 Set 7]. Disponível em: http:// tabnet.datasus.gov.br/cgi/deftohtm.exe?sinannet/cnv/ sifilisgestanters.def

11. Brasil. Ministério da Saúde (MS). Departamento de Atenção Básica (DAB). Histórico de Cobertura da Saúde da Família. [acessado 2016 Abr 23]. Disponível em: http://dab.saude.gov.br/portaldab/historico_cobertura_sf.php

12. StataCorp. Stata Statistical Software [computer program]. Version 13. College Station: StataCorp LP; 2013.
13. Kupek E, Oliveira JF. Transmissão vertical do HIV, da sífilis e da hepatite B no município de maior incidência de AIDS no Brasil: um estudo populacional no período de 2002 a 2007. Rev bras epidemiol 2012; 15(3):478-487.

14. Agência Nacional de Vigilância Sanitária (ANVISA). Avaliação em serviços de saúde: indicadores - coeficiente de letalidade. [acessado 2016 Abr 25]. Disponível em: http://www.anvisa.gov.br/servicosaude/avalia/indicadores/cl.htm

15. Instituto Brasileiro de Geografia e Estatística (IBGE). Divisão Política Administrativa: microregiões. [acessado 2016 Abr 9]. Disponível em: http://www.ngb.ibge.gov. br/Default.aspx?pagina $=$ micro

16. Duarte-Cunha M, Souza-Santos R, Matos HJD, Oliveira MLWD. Aspectos epidemiológicos da hanseníase: uma abordagem espacial. Cad Saude Publica 2012; 28(6):1143-1155.

17. Martins-Melo F, Lima M, Alencar C, Ramos A, Costa Carvalho F, Machado M, Heukelbach J. Tendência temporal e distribuição espacial do aborto inseguro no Brasil, 1996-2012. Rev Saude Publica 2014; 48(3):508520

18. Instituto de Pesquisa Econômica Aplicada (IPEA). Malhas. [acessado 2014 Set 8]. Disponível em: http://www. ipea.gov.br/ipeageo/malhas.html

19. Brasil. Ministério da Saúde (MS), Fundação Oswaldo Cruz (Fiocruz). Introdução a Estatística Espacial para Saúde Pública. Brasília: MS; 2007. (Série B. Textos Básicos de Saúde). (Série Capacitação e Atualização em Geoprocessamento em Saúde; 3).

20. Instituto Nacional de Pesquisas Espaciais (INPE). Terraview [computer program]. Versão 4.2.2. Brasília: INPE; 2013.

21. Coutinho KMV, Rizol PMSR, Nascimento LFC, Medeiros APPD. Fuzzy model approach for estimating time of hospitalization due to cardiovascular diseases. Cien Saude Colet 2015; 20(8):2585-2590.

22. Rufino R, Gracie R, Sena A, de Freitas CM, Barcellos C. Surtos de diarreia na região Nordeste do Brasil em 2013, segundo a mídia e sistemas de informação de saúde-Vigilância de situações climáticas de risco e emergências em saúde. Cien Saude Colet 2016; 21(3):777-788.

23. Azevedo AJP, Araújo AA, Ferreira MAF. Consumo de ansiolíticos benzodiazepínicos: uma correlação entre dados do SNGPC e indicadores sociodemográficos nas capitais brasileiras. Revista Cien Saude Colet 2016; 21(1):83-90.

24. Ferreira DB, Mattos IE. Tendência da mortalidade por câncer de mama em mulheres no estado do Rio de Janeiro, Brasil, 1996-2011. Cien Saude Colet 2015; 20(3):895-903. 
25. Pan American Health Organization (PAHO). Elimination of mother-to-child transmission of hiv and syphilis in the Americas: update 2015. Washington: PAHO; 2015.

26. Lima LHM, Gurgel MFC, Moreira-Silva SF. Avaliação da Sífilis Congênita no estado do Espírito Santo. DST J bras Doenças Sex Transm 2006; 18(2):113-116.

27. Reyna-Figueroa J, Esparza-Aguilar M, Hernández -Hernández LC, Fernández-Canton S, Collada VLRL. Congenital syphilis, a reemergent disease in Mexico: its epidemiology during the last 2 decades. Sex Transm Dis 2011; 38(9):798-801.

28. Alzate-Granados JP, Sánchez-Bello NF, Amaya-Arias AC, Peralta-Pizza F, Eslava-Schmalbach J. Disparidades en la incidencia de sífilis congénita en Colombia 2005 a 2011: Un estudio ecológico. Rev de Salud Publica 2012; 14(6):965-977.

29. Ortiz-Lopez N, Diez M, Diaz O, Simon F, Diaz A. Epidemiological surveillance of congenital syphilis in Spain, 2000-2010. Pediatr infect Dis J 2012; 31(9):988990.

30. Hong FC, Yang YZ, Liu XL, Feng TJ, Liu JB, Zhang CL, Lan LN, Yao MZ, Zhou H. Reduction in mother-tochild transmission of syphilis for 10 years in Shenzhen, China. Sex Transm Dis 2014; 41(3):188-193.

31. Mungrue K, Edwards J, Fyzul A, Bhoodhai B, Narinesingh A, Nanal S. Towards the Elimination of Syphilis in a Small Developing Country. J Sex Transm Dis 2015; 2015(1):1-5.

32. Ramiandrisoa J, Aubert L, Lespine EB, Alessandri JL, Robillard PY, Bertsch M, Gallay A, Goulet V, D’ortenzio E. Congenital Syphilis, Réunion Island, 2010 [carta]. Emerg Infec Dis 2011; 17(11):2082-2083.

33. Simms I, Tookey PA, Goh BT, Lyall H, Evans B, Townsend CL, Fifer H, Ison C. The incidence of congenital syphilis in the United Kingdom: February 2010 to January 2015. BJOG [periódico na Internet] 2016 Mar [acessado 2016 Set 9]; 67(2):[cerca de 6 p.]. Disponível em: http://onlinelibrary.wiley.com/ doi/10.1111/1471-0528.13950/pdf

34. Buffolano W, Agnese M, Pizzuti R. Secular trend on congenital infections: insights from Campania region register for perinatal infection, southern Italy. J Matern Neonatal Med 2011; 24(Supl. 1):94-96.

35. Peterman TA, Su J, Bernstein KT, Weinstock H. Syphilis in the United States: on the rise? [special report] Expert Rev Anti Infect Ther 2015; 13(2):161-168.

36. Su JR, Brooks LC, Davis DW, Torrone EA, Weinstock HS, Kamb ML. Congenital syphilis: trends in mortality and morbidity in the United States, 1999 through 2013. Am J Obstet Gynecol [periódico na Internet] 2016 Mar; [acessado 2016 Set 9] 214(3):[cerca de 9 p.]. Disponível em: http://www.ajog.org/article/S00029378(15)01270-3/pdf
37. Costa CC, Freitas LV, Souza DMN, Oliveira LL, Araújo ACM, Lopes MVO, Damasceno AKC. Sífilis congênita no Ceará: análise epidemiológica de uma década. Rev Esc Enferm USP 2013; 47(1):152-159.

38. Schetini J, Ferreira DC, Passos MRL, Salles EB, Santos DDG, Rapozo DCM. Estudo da prevalência de sífilis congênita em um hospital da rede SUS de Niterói - RJ. DST J bras Doenças Sex Transm 2005; 17(1):18-23.

39. Rio Grande do Sul. Secretaria do Planejamento, Mobilidade e Desenvolvimento Regional (SEPLAN) [Internet]. Índice de Desenvolvimento Humano - IDH e IDHM. [acessado 2016 Abr 11]. Disponível em: http:// www.atlassocioeconomico.rs.gov.br/upload/idh_ rs_2010.pdf

40. Venâncio TS, Tuan TS, Nascimento LFC. Incidência de tuberculose em crianças no estado de São Paulo, Brasil, sob enfoque espacial. Cien Saude Colet 2015; 20(5):1541-1547.

41. Brasil. Ministério da Saúde (MS). Portal da Saúde [Internet]. Brasil e Uruguai regulamentam acesso a serviços de saúde em áreas de fronteira. [acessado $2016 \mathrm{Abr}$ 11]. Disponível em: http://portalsaude.saude.gov.br/ index.php/cidadao/principal/agencia-saude/noticias -anteriores-agencia-saude/3997-brasil-e-uruguai-regulamentam-acesso-a-servicos-de-saude-em-areasde-fronteira

42. Bontempo CGC, Nogueira VMR, Fagundes HS. Atenção básica à saúde na fronteira Brasil-Uruguai: um olhar a partir dos gestores. Cad Ibero-Am Direito Sanit 2013; 2(2):897-907.

Artigo apresentado em 09/05/2016

Aprovado em 15/09/2016

Versão final apresentada em 17/09/2016 
